\title{
Veterinary vaccines against Toxoplasma gondii
}

\author{
Elisabeth A Innes/ ${ }^{+}$, Paul M Bartley, Stephen Maley, Frank Katzer, David Buxton
}

Moredun Research Institute, Pentlands Science Park, EH26 OPZ Bush Loan, Edinburgh

Toxoplasma gondii has a very wide intermediate host range and is thought to be able to infect all warm blooded animals. The parasite causes a spectrum of different diseases and clinical symptoms within the intermediate hosts and following infection most animals develop adaptive humoral and cell-mediated immune responses. The development of protective immunity to $\mathrm{T}$. gondii following natural infection in many host species has led researchers to look at vaccination as a strategy to control disease, parasite multiplication and establishment in animal hosts. A range of different veterinary vaccines are required to help control $\mathrm{T}$. gondii infection which include vaccines to prevent congenital toxoplasmosis, reduce or eliminate tissue cysts in meat producing animals and to prevent oocyst shedding in cats. In this paper we will discuss some of the history, challenges and progress in the development of veterinary vaccines against $\mathrm{T}$. gondii.

Key words: Toxoplasma gondii - veterinary - vaccine

Toxoplasma gondii has a very wide host range and is thought to be able to infect all warm blooded animals making it a highly successful parasitic organism. There is also a wide spectrum of disease manifesting within the various host species and different components contribute to the severity of the disease including host species, immune status of host and biological and genetic variation within the parasite (Innes 1997). T.gondii can cause acute fatal infection in some host species such as marsupials and new world monkeys that have evolved largely away from the cat (the definitive host of $T$. gondii) and have therefore not developed resistance to the parasite (Dubey \& Beattie 1988). In farm livestock species such as sheep and goats, congenital infection is common which may result in abortion and neonatal mortality (Buxton 1998). Animals bred to produce meat for human consumption may be persistently infected with $T$. gondii, contained within tissue cysts in the muscles and viscera and can act as important sources of infection for people. A very important animal in the life cycle of $T$. gondii and the epidemiology of the disease is the cat. Following a primary infection, cats will shed millions of oocysts in their faeces that can survive for 12-18 months in the environment, depending on climactic conditions, and are an important source of infection for grazing animals (Tenter et al. 2000). Therefore a range of different veterinary vaccines are required to help control $T$. gondii infection which include vaccines to prevent congenital toxoplasmosis, reduce or eliminate tissue cysts and to prevent oocyst shedding (Innes \& Vermeulen 2006). In this paper we will discuss some of the history, challenges and progress in the development of veterinary vaccines against $T$. gondii.

Financial support: Rural and Environment Research, Analysis Directorate, Scottish Government

+ Corresponding author: lee.innes@moredun.ac.uk

Received 10 October 2008

Accepted 3 December 2008

\section{Vaccines to prevent congenital toxoplasmosis}

Toxoplasma was first recognised to be an important pathogen in livestock species following reports from New Zealand describing $T$. gondii organisms in placental tissue from aborting sheep and within an aborted ovine foetus (Hartley et al. 1954, Hartley \& Marshall, 1957). The authors initially described this as New Zealand type II abortion and subsequently there were other reports of a similar disease in sheep occurring in other countries worldwide including Australia, UK and Europe. At the time ovine toxoplasmosis was first described in New Zealand, the route of transmission was still unclear. As sheep are herbivores it suggested that there may be another as yet undiscovered route of transmission that did not involve congenital transmission or the consumption of $T$. gondii tissue cysts within infected meat. The discovery in the late 1960's that cats could shed a new form of the parasite in their faeces that was very stable in the environment (Hutchison 1965) led to the recognition of the cat as the definitive host of the parasite (Frenkel et al. 1970, Hutchison et al. 1970, Ferguson et al. 1974) and the oocyst as a major source of infection for both animals and people, as well as being an important source of environmental contamination. The discovery of $T$. gondii occysts helped to explain transmission of infection to herbivores and therefore how the disease was spread within and between flocks of grazing animals. Ovine toxoplasmosis occurs in temperate sheep rearing countries worldwide where the climatic conditions favour oocyst survival (Buxton \& Rodger 2008). In 2007, the population of sheep and goats in the EU reached 108,9 million (http://epp.eurostat.ec.europa.eu) and while the incidence of toxoplasmosis is difficult to define with accuracy, a study in UK suggested that the disease was responsible for $1-2 \%$ of neonatal losses per annum (Blewett \& Trees 1987). If this incidence was extrapolated throughout Europe, this would mean a loss of around 1,5-2 million animals.

Young cats tend to become infected with $T$. gondii when they go hunting for the first time and eat wild ro- 
dents and birds. Around three-10 days after infection, cats start to shed oocysts for two-three weeks (Dubey $\&$ Beattie 1988). Each infected cat may shed 100 million oocysts into the environment and as few as 200 sporulated oocysts can cause congenital disease in naive sheep (McColgan et al. 1988). Infection in sheep is associated with contamination of feed or grazing land with sporulated oocysts (Plant et al. 1974, Faull et al. 1986). There is also an increasing likelihood of seroprevalence related to age of the animal indicating that there is extensive environmental contamination with oocysts and that most infections in sheep occur following birth (Waldeland 1977, Lunden et al. 1994). Some recent data suggests that in some circumstances persistently infected sheep may transmit the parasite to the foetus in subsequent pregnancies (Duncanson et al. 2001, Morley et al. 2005). These observations are interesting, although do appear to be uncommon. A further more complete study provided confirmatory evidence that there does not appear to be significant congenital transmission of T. gondii from persistently infected sheep to their offspring (Rodger et al. 2006). Clinical symptoms in sheep include foetal death, production of a mummified foetus, still born lamb or birth of a live but weak lamb (Buxton \& Rodger 2008). A significant factor in determining severity of disease is the stage of gestation when infection occurs, the earlier in gestation the more severe the consequences for the developing foetus (Watson \& Beverley 1971, Hartley \& Moyle 1974, Blewett et al. 1982).

In a naïve sheep, following ingestion of oocysts, the parasites excyst in the gut releasing sporozoites which are able to actively invade and multiply within the gut cells. The tachyzoite stage of the parasite multiplies asexually by a process of endodyogeny within a parasitophorous vacuole and then the parasites eventually rupture from the cell and go on and invade further cells (Lingelbach \& Joiner 1998). By day four following infection, tachyzoites may be found in the mesenteric lymph node (Dubey 1984) and the parasites are also found in the circulation where they can spread throughout the host (Wastling et al. 1993). In the pregnant animal, the tachyzoites invade and multiply within the caruncular septa in the placentome and then go on and invade the adjacent foetal trophoblast cells where they can spread to the rest of the foetus (Buxton \& Finlayson 1986). If infection of the foetus occurs early in gestation, while the foetal immune system is still relatively immature foetal death is the usual outcome, whereas infection later in gestation may result in the lamb being born live, but infected and immune. A Toxoplasma infection occurring at mid-gestation typically results in a stillborn or weak lamb accompanied by a small mummified foetus. Characteristic lesions on the infected placenta are white spots visible to the naked eye due to areas of necrosis resulting in the impaired function of the placenta (Buxton \& Rodger 2008). The natural immunomodulation, that occurs during pregnancy in the ewe acts to damp down inflammatory Th1-type immune responses within the placenta (Entrican 2002, Innes \& Vermeulen 2006), as a result placental tissue is particularly vulnerable to invasion and establishment of $T$. gondii tachyzoites. Following in- vasion of the host by the parasite the innate and adaptive immune responses are activated and act together to limit tachyzoite multiplication (Innes \& Vermeulen 2006).

Studies in sheep looking at the kinetics of development of in-vivo immune responses during a primary infection with $T$. gondii has shown the early induction of interferon gamma (IFN $\gamma$ ) within $48 \mathrm{~h}$ (Innes et al. 1995b). This early induction of IFN $\gamma$ may be important in limiting intracellular multiplication of the parasite (Oura et al. 1993) and may also help to create an appropriate cytokine microenvironment to induce a Th-1 type adaptive immune response (Gazzinelli et al. 1996). A phenotypic analysis of the lymphoblast cells responding to a primary T. gondii infection in sheep showed an initial predominance of CD4+ T-cells (Innes et al. 1995a). Around day 10 post-infection, when the peak lymphoblast response was observed, there was a switch to CD8+ T-cells being the dominant cell population. In vitro studies showed that these activated CD8+ T-cells were able to inhibit intracellular multiplication of tachyzoites (Innes et al. 1995a) and following the peak lymphoblast response in efferent lymph draining the site of infection the parasites were no longer detected within the lymph samples (Innes $\&$ Wastling 1995). Specific antibodies to T. gondii were detected from day 10-12 after the initial infection (Innes $\&$ Wastling 1995). Therefore it would appear that cellmediated immune responses involving IFN $\gamma, \mathrm{CD} 4+$ and $\mathrm{CD} 8+\mathrm{T}$-cells are involved in protective immunity and recovery from a primary infection, while specific antibody may be more important in defending the host following a secondary challenge (Innes \& Vermeulen 2006).

Following a primary infection with $T$. gondii, most sheep develop protective immunity against further disease therefore controlling the disease using vaccination may be a feasible goal. Current knowledge of protective immunity would suggest that an effective vaccine would have to stimulate cell-mediated immune responses. Attempting to induce such responses with different vaccine preparations would require an awareness of the role of regulatory cytokines in balancing and controlling the potential immunopathology caused by a vigorous inflammatory response at the materno-foetal interface (Innes \& Vermeulen 2006, Pfaff et al. 2007). Another challenge would be to devise methods of antigen delivery that would allow the processing and presentation of antigens within the correct MHC background in order to effectively stimulate the required T-cell responses. This is easier to achieve using a live vaccine preparation and may help to explain the success of the S48 strain vaccine used successfully to protect sheep and goats against congenital toxoplasmosis (O'Connell et al. 1988, Buxton \& Innes 1995).

\section{S48 strain Toxovax ${ }^{\circledR}$ vaccine}

The S48 strain was originally isolated from an aborted lamb in New Zealand and was maintained in the laboratory by repeated passage in mice. Researchers then observed that the parasites had over time lost the ability to differentiate into bradyzoites or oocysts and had become effectively an incomplete strain (O'Connell et al. 1988). The S48 strain tachyzoites induce a short-lived infection in sheep of around 14 days before being eliminated 
from the host by the sheep's immune system (Innes \& Wastling 1995). As this strain has lost the ability to differentiate into bradyzoites, it is not able to establish a persistent infection in the animal (Buxton 1993). It is also unable to initiate the sexual life cycle in cats and cannot lead to the production of oocysts. The S48 strain tachyzoites are able to undergo limited multiplication within the sheep, and therefore induce protective cellmediated immune responses involving $\mathrm{CD} 4+, \mathrm{CD} 8+$ T-cells and IFN $\gamma$ (Innes et al. 1995a). In sheep vaccinated with S48 and then challenged with an additional live infection, the parasite is prevented from spreading within the lymph system (Buxton et al. 1994). Therefore when a vaccinated pregnant sheep ingests oocysts on pasture the released sporozoites will invade the gut wall and enter the mesenteric lymph node where the immune system will be primed to limit the spread of the parasite from the lymph to the circulation of the host. Therefore the parasite will be prevented from reaching the placenta and causing disease and in addition there will be little dissemination of the parasite to other tissues. As a consequence, the vaccine may have the additional benefit of not only preventing congenital toxoplasmosis, but in addition reducing the risk of meat from the sheep harbouring $T$. gondii tissue cysts. A further advantage of this vaccine is that one shot will protect animals against T. gondii induced abortion for up to at least 18 months (Buxton et al. 1993). The vaccine is normally administered prior to mating and there is a meat and milk withdrawal period of six weeks following S48 vaccination. Disadvantages are that as the vaccine is live it has a relatively short shelf life and care has to be taken with administration as it is a zoonotic pathogen. Clearly, a live vaccine would not be appropriate for use in humans and those working on vaccines targeted for human use have focussed on identification of immunodominant antigens and effective vaccine delivery systems to induce the appropriate protective immune responses. These studies have largely involved the use of mouse models of congenital infection to test the efficacy of the different preparations. Pregnant sheep may be a very useful animal model to test the efficacy of different vaccine preparations to protect against congenital toxoplasmosis as the disease in sheep is very similar to that seen in pregnant women (Innes \& Vermeulen 2006).

\section{Vaccines to reduce tissue cysts}

A recent European multicentre study indicated that up to $60 \%$ of $T$. gondii infections may be attributed to the consumption of undercooked or cured meat products from animals infected with the parasite (Cook et al. 2000). The numbers of tissue cysts and their location varies between different meat producing livestock and the husbandry practices used to rear them. Meat from pigs, sheep, goats and free range poultry are thought to be the major sources of human infection (Tenter et al. 2000). Undercooked pork in particular was highlighted as a major risk to consumers in a study conducted in the USA looking at the presence of viable $T$. gondii within meat samples taken from retail meat stores (Dubey et al. 2005). Indoor reared pigs and poultry with adequate measures of hygiene and confinement have led to a decrease in the levels of $T$. gondii infection (Tenter et al. 2000). However, the rise in consumer demand for outdoor reared animal friendly production systems has led to a higher prevalence of $T$. gondii in these animals which may pose an increased risk to people consuming outdoor reared meat (Kijlstra et al. 2004). Development of vaccines to prevent or reduce $T$. gondii tissue cysts in meat producing animals would be of great benefit in reducing the risk of transmission to people. Animals grazing on pastures such as sheep and goats often show high seroprevalences in many parts of the world indicating that there is a high level of environmental contamination with T. gondii oocysts (Tenter et al. 2000) and tissue cysts are readily found in sheep muscles and tissues (Esteban et al. 1999).

The potential of the S48 Toxovax $^{\circledR}$ vaccine in reducing the development of tissue cysts in vaccinated sheep has been discussed above. A similar type of live vaccination approach using the RH strain of $T$. gondii had been used to immunise pigs to help prevent the development of parasite cysts in their tissues (Dubey et al. 1991). A further study improved the efficacy of immunisation with RH strain tachyzoites by using oligodeoxynucleotides containing immunostimulatory $\mathrm{CpG}$ motifs as an adjuvant (Kringel et al. 2004). The live vaccination approach using RH tachyzoites appeared to be more effective in prevention of $T$. gondii tissue cyst formation in pigs compared to a study using a crude fraction of $T$. gondii rhoptry proteins incorporated into immunostimulating complexes (Garcia et al. 2005). A recent study examined whether it was feasible to induce protective humoral and cell mediated immune responses in pigs following DNA vaccination (Jongert et al. 2008). Pigs were immunised intradermally using a GRA1-GRA7 DNA vaccine cocktail which induced strong humoral and type I cell-mediated immune responses including IFN $\gamma$ which is known to be important in inhibiting multiplication of T. gondii (Oura et al. 1993). These results were encouraging in the development of vaccines that may protect against the formation of $T$. gondii tissue cysts in meat producing animals.

\section{Vaccines to reduce oocyst shedding}

Following a primary infection with $T$. gondii cats will shed millions of oocysts in their faeces (Dubey \& Beattie 1988). Following this primary shedding of oocysts, cats do develop some immunity and therefore are unlikely to shed further oocysts upon re-infection or reshed oocysts from the original infection. However this immunity may not last for the lifetime of the cat and research has shown that cats may shed further oocysts when re-challenged around six years after their primary infection (Dubey 1995). These sporulated oocysts are major environmental contaminants and are an important source of infection for livestock and other animals including humans. There have been recent reports of human outbreaks of toxoplasmosis attributed to oocyst contamination of water supplies in Canada (Bowie et al. 1997) and Brazil (Bahia-Oliveira et al. 2003). In addition, there have been several reports of $T$. gondii oocysts 
from the environment contaminating oceans and being a source of fatal infection in marine mammals as these animals has very little resistance to the parasite (Dubey et al. 2003, Conrad et al. 2005). A vaccine to help prevent oocyst shedding in cats would be highly desirable and of significant benefit in limiting the environmental contamination with the parasite thus reducing infection in many intermediate hosts.

A promising candidate vaccine in this respect is T-263 strain of T. gondii (Frenkel et al. 1991). This mutant strain of $T$. gondii will only undergo partial development in the gut of the cat which does not result in production of oocysts. In addition, cats fed T-263 strain bradyzoites developed significant immunity against oocyst shedding following challenge with a complete strain of $T$. gondii (Frenkel et al. 1991). A further study showed improved efficacy of the vaccine when intact tissue cysts and released bradyzoites were administered in two oral doses to a group of cats (Freyre et al. 1993). The cats in this study were challenged 47 days later using a complete strain of T. gondii, T-265 and no oocyst shedding was detected in the cats after challenge (Freyre et al. 1993). The potential of this vaccine in the field was tested in a large scale trial conducted on young cats caught and trapped on eight commercial pig farms in the USA. The cats were vaccinated with the T-263 vaccine and the efficacy of the vaccine was measured indirectly by examining seroprevalence of other intermediate animal hosts, including the farmed pigs within the study farms (Mateus-Pinilla et al. 1999). The results showed a decrease in seroprevalence within the pig population implying that vaccinating the cats had reduced the shedding of $T$. gondii oocysts into the environment and therefore reduced the source of infectious material for the intermediate animal hosts in this area (Mateus-Pinilla et al. 1999). Further analysis of this study showed that the decrease in $T$. gondii seroprevalence observed in the farm pigs was related to the number of cats on the farm, oocyst survival in the environment and the vaccination of cats with the T-263 vaccine (Mateus-Pinilla et al. 2002).

The T-263 mutant strain has proved to have good efficacy in vivo, although a disadvantage for large scale production of the vaccine is that it is produced by infecting mice and the T. gondii cysts are harvested from the mouse brains. The vaccine is administered live therefore has a limited shelf life and may be hazardous to those administering it. The vaccine is kept frozen until delivery to try and maintain viability of the bradyzoites (Choromanski et al. 1995).

Other studies have looked at immunization of cats using different strains of $T$. gondii. In one study, kittens were vaccinated with $60 \mathrm{Co}$ irradiated tachyzoites of the Beverley strain of $T$. gondii which afforded partial protection against oocyst shedding when the kittens were challenged with $T$. gondii cysts from the Beverley strain (Omata et al. 1996). In the same study kittens vaccinated with 60Co irradiated or fixed tachyzoites of the RH strain, shed oocysts when challenged with the Beverly strain implying that development of protective immunity using the Beverley strain of $T$. gondii seemed to be specific to the immunising strain (Omata et al. 1996). In a further study, eight out of nine cats inoculated with ME49 strain of $T$. gondii were protected against oocyst shedding following challenge with three different strains of Toxoplasma, showing that the ME-49 strain was capable of inducing well cross protective immunity (Freyre et al. 2007). The ROP 2 antigen of $T$. gondii has been tested as a vaccine in cats using a recombinant feline herpesvirus type 1 vector to deliver the antigen, resulting in reduced numbers of cerebral parasites (Mishima et al. 2002). Most of the studies involving vaccination against $T$. gondii in cats have involved live vaccine preparations as it is likely that these will induce appropriate protective cell-mediated immune responses, however they do have drawbacks in terms of safety, large scale production and short shelf life.

Toxoplasma is a highly successful parasitic organism that is able to infect and live within a very wide range of different animal hosts. Improved understanding of disease pathogenesis within different host species along with knowledge of the parasite lifecycle and transmission routes has allowed more specific targeting of control and intervention strategies. Vaccination is an attractive option to control disease and to limit spread of the parasite both within the host and into the environment as most animals develop protective immune responses following primary infection with $T$. gondii. As $T$. gondii is an obligate intracellular parasite, cell-mediated immune responses involving both the innate and adaptive immune responses such as NK cells, CD4+ and CD8+ T-cells and both pro-inflammatory and regulatory cytokines are known to be important in protecting the host and limiting multiplication of the parasite. It is considerably easier to stimulate appropriate cell-mediated immune responses through live vaccine preparations as the relevant antigens are processed and presented to the host in a similar way to natural infection. This may explain why many of the successful veterinary vaccines to date are using live attenuated strains of the parasite that will undergo limited multiplication within the host, but not cause disease or persistent infection. However, live vaccines have drawbacks in that they can be problematic to produce in large quantities, they may be unstable, have a short shelf life and there are safety concerns due to the zoonotic nature of the parasite. In addition, live vaccines would not be considered safe to use in people. Therefore, the focus of current research is to identify the main protective antigens within the different life cycle stages of T. gondii and use novel antigen delivery systems to try and stimulate protective cell-mediated immune responses. Animal models of toxoplasmosis are very important to help us to generate the technology required to develop safe and effective vaccines against toxoplasmosis that could be used in humans.

\section{REFERENCES}

Bahia-Oliveira LM, Jones JL, Zevedo-Silva J, Alves CC, Orefice F, Addiss DG 2003. Highly endemic, waterborne toxoplasmosis in North Rio de Janeiro state, Brazil. Emerg Infect Dis 9: 55-62.

Blewett DA, Miller JK, Buxton D 1982. Response of immune and susceptible ewes to infection with Toxoplasma gondii. Vet Rec 28: 175-178. 
Blewett DA, Trees AJ 1987. The epidemiology of ovine toxoplasmosis with especial respect to control. Br Vet J Mar 143: 128-135.

Bowie WR, King AS, Werker DH, Isaac-Renton JL, Bell A, Eng SB, Marion SA 1997. Outbreak of toxoplasmosis associated with municipal drinking water. Lancet 350: 173-177.

Buxton D 1993. Toxoplasmosis: the first commercial vaccine. Parasitol Today 9: 335-337.

Buxton D 1998. Protozoan infections (Toxoplasma gondii, Neospora caninum and Sarcocystis spp.) in sheep and goats: recent advances. Vet Res 29: 289-310.

Buxton D, Finlayson J 1986. Experimental infection of pregnant sheep with Toxoplasma gondii: pathological and immunological observations on the placenta and foetus. J Comp Pathol 96: 319-333.

Buxton D, Innes EA 1995. A commercial vaccine for ovine toxoplasmosis. Parasitol 110 (Suppl.): S11-S16.

Buxton D, Rodger SM 2008. Toxoplasmosis and neosporosis. In Diseases of sheep. 4th ed (Aitken ID ed) Wiley-Blackwell, Hoboken, p. 112-118.

Buxton D, Thomson KM, Maley S, Wastling JM, Innes EA, Panton WRM 1994. Primary and secondary responses of the ovine lymph node to Toxoplasma gondii: cell output in efferent lymph and parasite detection. J Comp Pathol 111: 231-241.

Buxton D, Thomson KM, Maley S, Wright S, Bos HJ 1993. Experimental challenge of sheep 18 months after vaccination with a live (S48) Toxoplasma gondii vaccine. Vet Rec 133: 310-312.

Choromanski L, Freyre A, Popiel R, Brown K, Grieve R, Shibley G 1995. Safety and efficacy of modified live feline Toxoplasma gondii vaccine. Dev Biol Stand 84: 269-281.

Conrad PA, Miller MA, Kreuder C, James ER, Mazet J, Dabritz H, Jessup DA, Gulland F, Grigg ME 2005. Transmission of Toxoplasma: clues from the study of sea otters as sentinels of Toxoplasma gondii flow into the marine environment. Int J Parasitol 35: $1155-1168$

Cook AJ, Gilbert RE, Buffolano W, Zufferey J, Petersen E, Jenum PA, Foulon W, Semprini AE, Dunn DT 2000. Sources of Toxoplasma infection in pregnant women: European multi centre case-control study. European Research Network on Congenital Toxoplasmosis. $B M J 15: 142-147$.

Dubey JP 1984. Experimental toxoplasmosis in sheep fed Toxoplasma gondii oocysts. Int Goat Sheep Res 2: 93-104.

Dubey JP 1995. Duration of immunity to shedding of Toxoplasma gondii oocysts by cats. $J$ Parasitol 81 : 410-415.

Dubey JP, Beattie CP 1988. Toxoplasmosis of animals and man, Boca Raton, Florida, 220 pp.

Dubey JP, Hill DE, Jones JL, Hightower AW, Kirkland E, Roberts JM, Marcet PL, Lehmann T, Vianna MCB, Sreekumar C, Kwok OCH, Gamble HR 2005. Prevalence of viable Toxoplasma gondii in beef, chicken and pork from retail meat stores in the United States: risk assessment to consumers. J Parasitol 91: 1082-1093.

Dubey JP, Urban JF Jr, Davis SW 1991. Protective immunity to toxoplasmosis in pigs vaccinated with a nonpersistent strain of Toxoplasma gondii. Am J Vet Res 52: 1316-1319.

Dubey JP, Zarnke R, Thomas NJ, Wong SK, Van Bonn W, Briggs M, Davis JW, Ewing R, Mensea M, Kwok OCH, Romand S, Thulliez P 2003. Toxoplasma gondii, Neospora caninum, Sarcocystis neurona and Sarcocystis canis-like infections in marine mammals. Vet Parasitol 116: 275-296.

Duncanson P, Terry RS, Smith JE, Hide G 2001. High levels of con- genital transmission of Toxoplasma gondii in a commercial sheep flock. Int J Parasitol 31: 1699-1703.

Entrican E 2002. Immune regulation during pregnancy and host-pathogen interactions in infectious abortion. J Comp Pathol 126: 79-94.

Esteban-Redondo I, Maley SW, Thomson K, Nicoll S, Wright S, Buxton D, Innes EA 1999. Detection of T. gondii in tissues of sheep and cattle following oral infection. Vet Parasitol 86: 155-171.

Faull WB, Clarkson MJ, Winter AC 1986. Toxoplasmosis in a flock of sheep: some investigations into its source and control. Vet Rec 119: 491-493.

Ferguson DJ, Hutchison WM, Dunachie JF, Sim JC 1974. Ultrastructural study of early stages of asexual multiplication and microgametogony of Toxoplasma gondii in the small intestine of the cat. Acta Pathol Microbiol Scand Microbiol Immunol 82: 167-181.

Frenkel JK, Dubey JP, Miller NL 1970. Toxoplasma gondii in cats: faecal stages identified as coccidian oocysts. Science 167: 893-896.

Frenkel JK, Pfefferkorn ER, Smith DD, Fishback JL 1991. Prospective vaccine prepared from a new mutant of Toxoplasma gondii for use in cats. Am J Vet Res 52: 759-763.

Freyre A, Choromanski L, Fishback JL, Popiel I 1993. Immunisation of cats with tissue cysts, bradyzoites and tachyzoites of the T-263 strain of Toxoplasma gondii. J Parasitol 79: 716-719.

Freyre A, Falcon J, Mendez J, Gastell T, Venzal JM 2007. Toxoplasma gondii cross-immunity against the enteric cycle. $J$ Parasitol 115: 48-52.

Garcia JL, Gennari SM, Navarro IT, Machado RZ, Sinhorini IL, Freire RL, Marana ER, Tsutsui V, Contente AP, Begale LP 2005. Partial protection against tissue cyst formation in pigs vaccinated with crude rhoptery proteins of Toxoplasma gondii. Vet Parasitol 129: 209-217.

Gazzinelli RT, Amichay D, Sharton-Kersten T, Grunwald E, Farber JM, Sher A 1996. Role of macrophage-derived cytokines in the induction and regulation of cell-mediated immunity to Toxoplasma gondii. In U Gross (ed.). Toxoplasma gondii, Springer-Verlag, Heidelberg, p. 127-139.

Hartley WJ, Jebson JL, McFarlane D 1954. New Zealand type II abortions in ewes. Aust Vet J 30: 216-218.

Hartley WJ, Marshall SC 1957. Toxoplasmosis as a cause of ovine perinatal mortality. $N Z$ Vet $J$ 5: 119-124.

Hartley WJ, Moyle GG 1982. Further observations on the epidemiology of ovine Toxoplasma infection. Aust J Exp Biol Med Sci 52: 647-653.

Hutchison WM 1965. Experimental transmission of Toxoplasma gondii. Nature 206: 961-962.

Hutchison WM, Dunachie JF, Sim JC, Work K 1970. Coccidian-like nature of Toxoplasma gondii. Br Med J 1: 142-144.

Innes EA 1997. Toxoplasmosis: comparative species susceptibility and host immune response. Comp Immunol Microbiol Infect Dis 20: 131-138.

Innes EA, Panton WR, Sanderson A, Thomson KM, Wastling JM, Maley SW, Buxton D 1995a. Induction of CD4+ and CD8+ T cell responses in efferent lymph responding to Toxoplasma gondii infection: analysis of phenotype and function. Parasite Immunol 17: $151-160$.

Innes EA, Panton WR, Thomson KM, Maley S, Buxton D 1995b. Kinetics of interferon gamma production in vivo during infection with the S48 vaccine strain of Toxoplasma gondii. J Comp Pathol 113: 89-94.

Innes EA, Vermeulen AN 2006. Vaccination as a control strategy 
against the coccidial parasites Eimeria, Toxoplasma and Neospora. Parasitol 133: 145-168.

Innes EA, Wastling JM 1995. Analysis of in vivo immune responses during Toxoplasma gondii infection using the technique of lymphatic cannulation. Parasitol Today 11: 268-271.

Jongert E, Melkebeek V, De Craeye S, Dewit J, Verhelst D, Cox E 2008. An enhanced GRA1-GRA7 cocktail DNA vaccine primes antiToxoplasma immune responses in pigs. Vaccine 26: 1025-1031.

Kijlstra A, Eissen OA, Cornelissen J, Munniksma K, Eijck I, Kortbeek T 2004. Toxoplasma gondii infection in animal-friendly pig production systems. Invest Ophthalmol Vis Sci 45: 3165-3169.

Kringel H, Dubey JP, Beshah E, Hecker R, Urban JF Jr 2004. CpGoligodeoxynucleotides enhance porcine immunity to Toxoplasma gondii. Vet Parasitol 123: 55-66.

Lingelbach K, Joiner KA 1998. The parasitophorous vacuole membrane surrounding Plasmodium and Toxoplasma: an unusual compartment in infected cells. J Cell Sci 111: 1467-1475.

Lunden A, Nasholm A, Uggla A 1994. Long-term study of Toxoplasma gondii infection in a Swedish sheep flock. Acta Vet Scand 35: $273-281$.

Mateus-Pinilla NE, Dubey JP, Choromanski L, Weigel RM 1999. A field trial of the effectiveness of a feline Toxoplasma gondii vaccine in reducing $T$. gondii exposure for swine. $J$ Parasitol 85: 855-860.

Mateus-Pinilla NE, Hannon B, Weigel RM 2002. A computer simulation of the prevention of the transmission of Toxoplasma gondii on swine farms using a feline T.gondii vaccine. Prev Vet Med 55: 17-36.

McColgan C Buxton D, Blewett D 1988. Titration of Toxoplasma gondii oocysts in non-pregnant sheep and the effects of subsequent challenge during pregnancy. Vet Rec 123: 467-470.

Mishima M, Xuan X, Yokoyama N, Igarashi I, Fujisaki K, Nagasawa H, Mikami T 2002. Recombinant feline herpesvirus type I expressing Toxoplasma gondii ROP2 antigen inducible protective immunity in cats. Parasitol Res 88: 144-149.
Morley EK, Williams RH, Hughes JM, Terry RS, Duncanson P, Hide G 2005. Significant familial differences in the frequency of abortion and Toxoplasma gondii infection within a flock of Charollais sheep. Parasitology 131(Pt. 2): 181-185.

O'Connell E, Wilkins MF, Te Punga WA 1988. Toxoplasmosis in sheep. II. The ability of a live vaccine to prevent lamb losses after an intravenous challenge with Toxoplasma gondii. N Z Vet J 36: 1-4.

Omata Y, Aihara Y, Kanda M, Saito A, Igarashi I, Suzuki N 1996. Toxoplasma gondii experimental infection in cats vaccinated with 60 Co-irradiated tachyzoites. Vet Parasitol 65: 173-183.

Oura CA, Innes EA, Wastling JM, Entrican G, Panton WR 1993. The inhibitory effect of ovine recombinant interferon-gamma on intracellular replication of Toxoplasma gondii. Parasite Immunol 15: 535-538.

Pfaff AW, Abou-Bacar A, Letscher-Bru V, Villard O, Senegas A, Mousli M, Candolfi E 2007. Cellular and molecular physiopathology of congenital toxoplasmosis: the dual role of IFN $\gamma$. Parasitol 134: 1895-1902.

Plant JW, Richardson N, Moyle GG 1974. Toxoplasma infection and abortion in sheep associated with feeding of grain contaminated with cat faeces. Aust Vet J 50: 19-21.

Rodger SM, Maley SW, Wright SE, Mackellar A, Wesley F, Sales J, Buxton D 2006. Ovine toxoplasmosis; the role of endogenous transmission. Vet Rec 159: 768-772.

Tenter AM, Heckeroth AR, Weiss LM 2000. Toxoplasma gondii: from animals to humans. Int J Parasitol 30: 1217-1258.

Waldeland H 1977. Toxoplasmosis in sheep. Influence of various factors on the antibody contents. Acta Vet Scand 18: 237-247.

Wastling JM, Nicoll S, Buxton D 1993. Comparison of two gene amplification methods for the detection of Toxoplasma gondii in experimentally infected sheep. J Med Microbiol 38: 360-365.

Watson WA, Beverley JKA 1971. Epizootics of toxoplasmosis causing ovine abortion. Vet Rec 88: 120-124. 